\title{
ESTIMATIVA DE FERRO BIODISPONÍVEL EM DIETAS DE CRECHES FILANTRÓPICAS DA CIDADE DE SÃO PAULO: CONSIDERAÇÕES SOBRE A FORTIFICAÇÃO DA FARINHA DE TRIGO COM FERRO
}

\author{
Edna H. S. Machadoํ, Cristiane H. Sales ${ }^{2}$, Anne L. D. Brasil ${ }^{3}$, Josefina A. P. Braga ${ }^{4}$, Célia Colli ${ }^{5}$
}

Objetivo: Estimar o ferro biodisponível nas dietas oferecidas às crianças de um a dois anos de idade, em creches filantrópicas do município de São Paulo, e, a partir dos cardápios avaliar o ferro considerando as alterações na concentração de ferro dietético pela fortificação das farinhas de trigo.

Métodos: O ferro total e o ferro biodisponível foram estimados nas quatro refeições diárias planejadas para um período de cinco dias, utilizando-se os cardápios padronizados adotados em oito creches filantrópicas do município de São Paulo. Considerando-se a concentração de ferro de cada dieta individual, bem como a de proteína animal e de vitamina C, calculou-se a quantidade de ferro biodisponível ofertada a cada refeição, utilizando-se a equação de Monsen e Balintfly, que possibilita essa estimativa, a partir da relação entre esses fatores. Estimou-se ainda, nas mesmas dietas, o ferro total e biodisponível e suas densidades, considerando-se o acréscimo na concentração de ferro não heme derivado da fortificação de farinha de trigo e de milho com 4,2 mg Fe/100 g. Para o cálculo da oferta de nutrientes das dietas, foi utilizado o Programa Nutwin ${ }^{\mathbb{R}}$, versão 1.5.

Resultados: A quantidade média diária de ferro e de ferro biodisponível oferecidos com as dietas foram de $6,2(0,8) \mathrm{mg} / \mathrm{d}$ e $0,53(0,06) \mathrm{mg} / \mathrm{d}$. Levando-se em conta a fortificação, esses valores aumentaram para, respectivamente, $7,7(0,9) \mathrm{mg} / \mathrm{d}$ e $0,59(0,07)$ $\mathrm{mg} / \mathrm{d}$. As médias de densidade de ferro e de ferro biodisponível com e sem fortificação, foram de, respectivamente, $5,8(1,3) \mathrm{mg} / 1.000 \mathrm{kcal}$ e $7,7(1,7) \mathrm{mg} / 1.000 \mathrm{kcal}$, e $0,42(0,10) \mathrm{mg} / 1.000 \mathrm{kcal} \mathrm{e} 0,49(0,13) \mathrm{mg} / 1.000 \mathrm{kcal}$.

Conclusão: Embora com a fortificação da farinha de trigo tenha havido aumento na concentração do ferro total nas dietas avaliadas $(24 \%$ ), a estimativa de ferro biodisponível ofertado mudou apenas $11 \%$. Para garantir o atendimento das necessidades diárias de 1,26 mg/d (percentil 97,5), considerando a biodisponibilidade de 9,0\% obtida nas dietas ofertadas a essas crianças, a recomendação deveria ser de cerca de $13 \mathrm{mg} / \mathrm{d}$, e não de $7 \mathrm{mg} / \mathrm{d}$. Esse nível de ingestão de ferro não poderia ser atingido somente com a fortificação das farinhas.

Palavras-chave: ferro, creches, dietas.

\section{ESTIMATION OF BIOAVAILABLE IRON IN PHILANTHROPIC DAY CARE CENTERS IN THE CITY OF SÃO PAULO: CONSIDERATIONS OVER IRON FORTIFICATION OF WHEAT FLOUR}

Objective: To assess the potential of bioavailable iron in the diets offered to children aged one to two years in day-care centers in the city of Sao Paulo, Brazil, and, from the diets to assess the iron considering the changes in the concentration of the dietary iron fortification of wheat flour.

Methods: The total iron offered and the bioavailable iron were assessed from four daily meals planned for the day-care centers, over a period of five days, using the planned meals for eight beneficent day-care centers of the Sao Paulo. From the iron concentration of each individual diet, the amounts of animal protein and vitamin C, the bioavailable iron offered was calculated, using the empiric equation of Monsen and Balintfly, from the relationship among these factors. It was estimated yet, in the same

\footnotetext{
1 Programa de Pós-Graduação em Nutrição Humana Aplicada - PRONUT, Departamento de Alimentos e Nutrição Experimental, Faculdade de Ciências Farmacêuticas, Universidade de São Paulo. São Paulo, SP. E-mail: ednama@usp.br

${ }^{2}$ Universidade de São Paulo, Faculdade de Ciências Farmacêuticas, Departamento de Alimentos e Nutrição Experimental. Av. Prof. Lineu Prestes, 580, Bl.14. CEP. 05.508-000, São Paulo,SP. E-mail: chsales@usp.br

${ }^{3}$ Universidade Federal de São Paulo - UNIFESP/EPM, Departamento de Pediatria. São Paulo, SP. E-mail : nutmet@terra.com.br

${ }^{4}$ Universidade Federal de São Paulo - UNIFESP/EPM. São Paulo, SP. E-mail: josefina.hped@pediatria.epm.br

${ }^{5}$ Universidade de São Paulo, Faculdade de Ciências Farmacêuticas, Departamento de Alimentos e Nutrição Experimental.Av. Prof. Lineu Prestes, 580, Bl. 14. CEP: 05.508-000 São Paulo,SP. E-mail: cecolli@usp.br
} 
meals, the total and bioavailable iron and your densities, considering the increase in the non-heme iron concentration derived from the fortification of the wheat and corn flours with $4.2 \mathrm{mgFe} / 100 \mathrm{~g}$. Nutwin ${ }^{\circledR}$ Software version 1.5 was used for the calculation of nutrient contents in the diets.

Results: The means of iron content and bioavailable iron in the daily diets were $6.2(0.8) \mathrm{mg} / \mathrm{d}$ and $0.53(0.06) \mathrm{mg} / \mathrm{d}$. Considering fortification, these means increased to $7.7(0.9) \mathrm{mg} / \mathrm{d}$ and $0.59(0.07) \mathrm{mg} / \mathrm{d}$, respectively. The means of Fe (dFe) and bioavailable Fe (dFebio) densities without and with flour fortification were $5.8(1.3) \mathrm{mg} / 1,000 \mathrm{kcal}$ and $7.7(1.7) \mathrm{mg} / 1,000 \mathrm{kcal}$, and $0.42(0.10)$ $\mathrm{mg} / 1,000 \mathrm{kcal}$ and $0.49(0.13) \mathrm{mg} / 1,000 \mathrm{kcal}$.

Conclusion: Although with iron fortification of the wheat flour there was an increase in the total iron in the diets assessed (24 \%), the amount of the estimated bioavailable iron changed only $11 \%$. To warrant the daily requirement of $1.26 \mathrm{mg} / \mathrm{d}$ ( $97.5 \mathrm{percentile})$, with $9.0 \%$ of estimated bioavilable iron the recommended iron intake should be $13 \mathrm{mg} / \mathrm{d}$, not $7.0 \mathrm{mg} / \mathrm{d}$. However, this level of daily ingested iron could not be achieved only with flour fortification.

Key words: iron, day care centers, diets.

\section{Introdução}

Nas últimas décadas, diversos países em desenvolvimento adotaram estratégias no combate a anemia ferropriva, que atinge principalmente populações com baixo poder aquisitivo, como medida para contornar os grandes prejuízos sociais e econômicos dessa deficiência nutricional [1].

No Brasil, embora não houvesse nenhuma pesquisa de âmbito nacional que levantasse a prevalência de anemia na população, inúmeros trabalhos desenvolvidos nos últimos vinte anos apontaram para uma prevalência de anemia variando, até 2004, entre 11 $\%$ e $89 \%$, em um dos principais grupos de risco, o préescolar [2-4].

A anemia por deficiência de ferro $(\mathrm{Fe})$ em préescolares no Brasil sempre esteve associada à má distribuição de renda e à falta de implementação de políticas públicas que priorizassem a assistência à saúde $\mathrm{e}$ nutrição e o saneamento básico [2,5]. Assim, os fatores que mais contribuem para a deficiência de Fe nesse grupo são: infecções, baixo peso ao nascer, crescimento rápido próprio dos lactentes, além do consumo de alimentos com Fe de baixa biodisponibilidade [2, 6].

A fortificação compulsória das farinhas de trigo e de milho com $\mathrm{FeSO}_{4}$ (4,2 mg Fe/100 g de farinha), implantada no Brasil em 2004, veio ao encontro da recomendação da Organização Mundial de Saúde OMS, de aumentar o aporte de Fe das dietas de préescolares e de adultos consumidores de pães, massas e biscoitos [7].

Por consumirem pequena quantidade desses alimentos, as crianças menores de dois anos, grupo de maior risco para a anemia ferropriva, são as menos atingidas por essa estratégia. Por esse motivo, o aumento da porcentagem de Fe biodisponível (FeBio) passa a ser importante instrumento de melhoria da qualidade do $\mathrm{Fe}$ dietético para esse grupo.

O Centro de Educação Infantil (creche) funciona como apoio às famílias nos aspectos educacionais, afetivos e alimentares fundamentais para as crianças. Para famílias de baixa renda, cujas mães permanecem fora de casa durante o dia, as refeições oferecidas nas creches podem ser suficientes para atender as necessidades mais imediatas das crianças, além de prevenir distúrbios nutricionais [8]. Szarfarc et al. [9], analisando a densidade de $\mathrm{Fe}$ em dietas de crianças de zero a 59 meses de idade do município de São Paulo, verificaram que a inadequação de Fe não decorria apenas de insuficiência energética, mas da composição dietética com alimentos de baixa biodisponibilidade do mineral. Assim, a garantia da oferta de alimentos com Fe de boa biodisponibilidade, bem como alimentos que aumentem essa biodisponibilidade do Fe seria imprescindível.

O FeBio pode ser avaliado a partir da aplicação de alguns algoritmos, dentre eles os propostos por Monsen e Balintfy [10]. São equações empíricas em que a resposta - \% de Fe biodisponível - é relacionada com os fatores estimuladores da absorção (proteína animal e ácido ascórbico) presentes nas diferentes refeições. O uso desses algoritmos no planejamento dos cardápios para crianças institucionalizadas permite maior segurança da adequação do Fe oferecido com a alimentação.

Assim, o objetivo deste trabalho foi estimar a quantidade de $\mathrm{Fe}$ biodisponível (FeBio) nas dietas oferecidas a crianças de um a dois anos de idade, em creches filantrópicas do município de São Paulo. Para tanto, considerou-se o acréscimo de Fe proveniente da fortificação das farinhas de trigo com o mineral, ao nível recomendado na legislação. 


\section{Material e Métodos}

Dietas

O estudo foi desenvolvido a partir dos cardápios padronizados de oito creches do município de São Paulo. As creches selecionadas, por conveniência, são mantidas por uma Instituição Filantrópica, que atende crianças de baixa renda, e que utiliza o mesmo padrão de alimentação para todas as refeições.

As creches avaliadas situam-se na zona central $(\mathrm{n}=2)$, no centro expandido $(\mathrm{n}=2)$, na zona leste $(\mathrm{n}=2)$ e na zona sul $(\mathrm{n}=2)$ do município de São Paulo, e, do total de 942 crianças atendidas, 133 delas tinham idades entre um e dois anos.

Foram avaliados, portanto, os cardápios de uma semana (cinco dias consecutivos) planejados para o grupo etário de um a dois anos. Esses cardápios se repetem ao longo do ano, com alterações nas preparações, mas não no grupo alimentar.

Como em todas as creches municipais e conveniadas com a Prefeitura do Município de São Paulo, a padronização per capita dos alimentos presentes nos cardápios das creches seguiu, até 2006, as diretrizes da atual Secretaria Municipal de Gestão [10].

Ao longo do dia foram oferecidos: desjejum (leite, pão e algum recheio ou bolo ou biscoito); almoço (arroz, feijão, carne, legume, saladas, doces ou frutas);
Onde \% FeNH Bio é a porcentagem de Fe não heme biodisponível, e EF é o somatório do total de carne (em gramas) e de vitamina C (em mg) de cada refeição. A equação foi aplicada para aquelas refeições em que EF $<75$ unidades. Quando EF $\geq 75$ unidades considerou-se a $\%$ de FeBio como $8 \%$, e, quando igual a zero, como $3 \%$.

O total de Fe heme biodisponível da dieta foi determinado considerando-se $23 \%$ de biodisponibilidade [10]. lanche (biscoito ou bolo e leite ou suco); e jantar (sopa, risoto ou macarronada). Uma vez na semana a mistura de arroz e feijão foi substituída por macarrão.

\section{Análise da oferta dietética}

A oferta dietética de energia, de macronutrientes, de vitamina $\mathrm{C}$ e de $\mathrm{Fe}$ das quatro refeições diárias foi calculada utilizando-se o Programa de Apoio à Nutrição, Nutwin ${ }^{\circledR}$, versão 1,5 [12]. A concentração de $\mathrm{Fe}$ heme nas dietas foi determinada assumindo-se que, do total de Fe da carne bovina, $57 \%$ é Fe heme, e do total de Fe da carne de frango, $25 \%$ [13]. Considerou-se como adequada a distribuição de 45 a 65 $\%$ do total de energia em carboidratos, 25 a $35 \%$ do total em lipídios e de 5 a $20 \%$ do total em proteínas [14].

Para estimar a necessidade de energia foi considerado o gasto energético basal para o estágio de vida estudado: $(89 \times$ peso $[\mathrm{kg}]-100)+20$ [14]. Para o cálculo, utilizou-se o peso correspondente ao percentil 50 para crianças de um ano e meio de idade. As ofertas de Fe total e de FeBio foram expressas em $\mathrm{mg} / \mathrm{d}$, e suas densidades em mg/1.000 kcal.

\section{Estimativa do Fe biodisponivel}

A estimativa de FeBio foi determinada por refeição (desjejum, almoço, lanche e jantar), a partir do total de $\mathrm{Fe}$ heme e não heme. $\mathrm{O} \mathrm{Fe}$ não heme biodisponível (FeNH Bio) foi obtido a partir da equação de Monsen e Balintfy [10]:

\section{, $93 \times \ln \left(\frac{\mathrm{EF}+100}{100}\right)$}

\section{Fe ofertado com a fortificação}

Para a obtenção da oferta de $\mathrm{Fe}$ dos alimentos à base de farinha fortificada (biscoitos, bolos, pães e macarrão), considerou-se o conteúdo de farinha de trigo em gramas $(70 \%$ nos biscoitos, $76 \%$ no macarrão e 81 $\%$ no pão) [15] e o nível de fortificação de 4,2 mg Fe/100 $\mathrm{g}$ de farinha, que é o estabelecido na legislação [7]. Identificados os alimentos e quantificado o total de Fe de adição nos alimentos com farinha de trigo, o total de $\mathrm{Fe}$ de adição foi somado, por refeição, ao total de Fe obtido nas refeições sem fortificação, obtendo-se, assim, o total de Fe das dietas com fortificação. 
Dentre os alimentos ofertados nos cardápios, nenhum tinha farinha de milho dentre os ingredientes.

Estimativa da evolução da prevalência de anemia em creches

Para estimar a evolução da prevalência de anemia em creches do município de São Paulo e da região Sudeste, da década de 90 até os dias atuais, foi feito levantamento bibliográfico em bases de dados nacionais, utilizando-se os descritores: creche, prevalência e anemia. Dentre os trabalhos identificados, foram selecionados os que utilizavam a mesma metodologia para determinar a concentração de hemoglobina de crianças até 59 meses (fotômetro portátil - HemoCue).

Aspectos éticos

O projeto foi aprovado pelo Comitê de Ética da Universidade Federal de São Paulo (CEP no 0733/02).

\section{Análise estatística}

O teste de Wilcoxon foi utilizado para comparar, inter-grupos, os parâmetros de $\mathrm{Fe}$ (Fe total,
FeBio, densidade de Fe e densidade de FeBio) avaliados nos cardápios planejados com e sem farinha fortificada com Fe.

Para comparação intra-grupo das médias de energia e dos parâmetros de $\mathrm{Fe}$ de cada refeição foi utilizado ANOVA e o teste de Tukey.

Para avaliar a correlação dos dados de prevalência de anemia foi utilizado o teste de Kolmogorov-Smirnov para verificar a normalidade dos dados, e depois a correlação de Pearson.

As análises foram realizadas no Software SPSS, versão $13.0{ }^{[16]}$, adotando-se nível de significância de $5 \%$.

\section{Resultados}

A distribuição de energia das dietas planejadas variou de 946 a $1.258 \mathrm{kcal} / \mathrm{d}$, com média de 1.112(81) $\mathrm{kcal} / \mathrm{d}$ (Figura 1), e a necessidade estimada de energia do grupo foi de $912 \mathrm{kcal} / \mathrm{kg} / \mathrm{d}$ [14]. A distribuição de energia correspondente aos macronutrientes foi adequada: $57 \%$ em carboidratos, $15 \%$ em proteínas e $28 \%$ em lipídios.

Figura 1. Energia em dietas planejadas para crianças de um a dois anos atendidas em creches assistenciais do município de São Paulo.

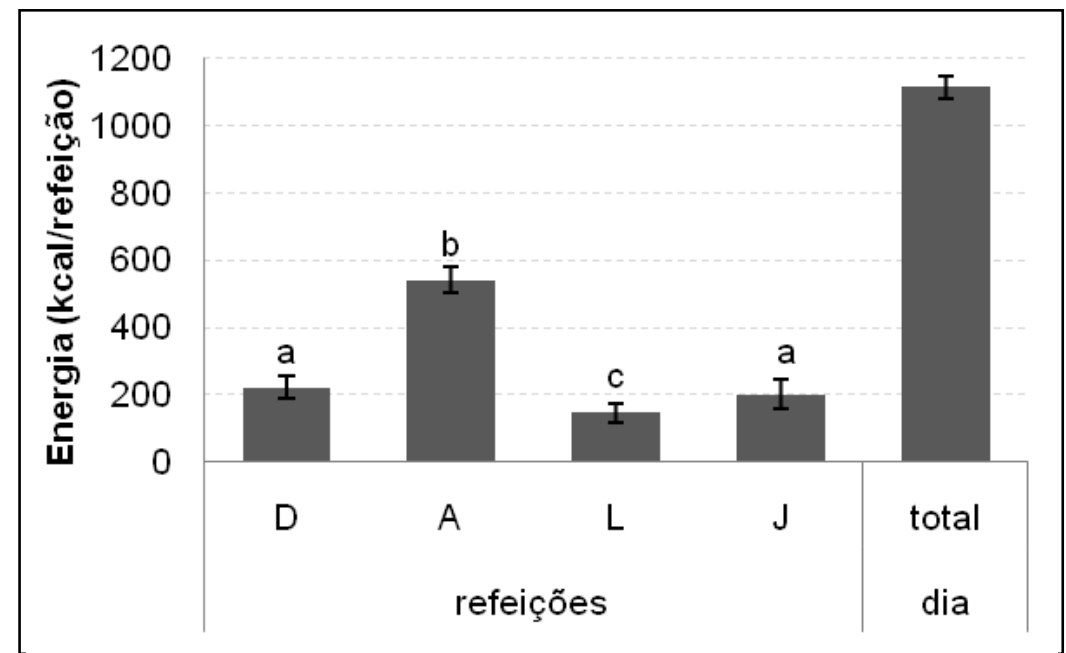

$\mathrm{D}=$ desjejum, $\mathrm{A}=$ almoço, $\mathrm{L}=$ lanche, $\mathrm{J}=$ jantar

A oferta de 6,15(0,76) $\mathrm{mg}$ de $\mathrm{Fe}$ total/d, não sendo considerada a fortificação da farinha de trigo, não atendia a meta de oferta de sete $\mathrm{mg} \mathrm{Fe} / \mathrm{d}$ [15].
Considerando-se a fortificação, a oferta de $7,7(0,9) \mathrm{mg}$ $\mathrm{Fe} / \mathrm{d}$, chegou a suplantar a RDA (Tabela 1). 
Tabela 1. Ferro total e estimativa de Fe biodisponível (FeBio) em dietas planejadas para crianças de um a dois anos, em creches assistenciais do município de São Paulo, com e sem fortificação com ferro da farinha de trigo.

\begin{tabular}{|c|c|c|c|}
\hline PARÂMETROS & SEM FORTIFICAÇÃO & $\begin{array}{c}\text { COM FORTIFICAÇÃO } \\
(4,2 \mathrm{mg} \mathrm{Fe} / 100 \mathrm{~g})\end{array}$ & $p^{*}$ \\
\hline $\mathrm{Fe} T(\mathrm{mg} / \mathrm{d})$ & $6,2(0,76)$ & $7,7(0,87)$ & 0,012 \\
\hline $\mathrm{Fe} \mathrm{H}(\mathrm{mg} / \mathrm{d})$ & $1,0(0,08)$ & $1,0(0,08)$ & 1,000 \\
\hline $\mathrm{Fe} \mathrm{NH}(\mathrm{mg} / \mathrm{d})$ & $5,2(0,73)$ & $6,7(0,86)$ & 0,012 \\
\hline $\mathrm{dFe}(\mathrm{mg} / 1000 \mathrm{kcal})$ & $5,8(1,25)$ & $7,7(1,65)$ & 0,012 \\
\hline Fe'TBio (mg/d) & $0,53(0,06)$ & $0,59(0,07)$ & 0,011 \\
\hline Fe HBio (mg/d) & $0,23(0,02)$ & $0,23(0,02)$ & 1,000 \\
\hline Fe NH Bio (mg/d) & $0,30(0,06)$ & $0,36(0,06)$ & 0,012 \\
\hline dFeBio (mg/1000kcal) & $0,42(0,10)$ & $0,49(0,13)$ & 0,012 \\
\hline$\%$ FeBio & $8,6(0,38)$ & $7,6(0,40)$ & 0,012 \\
\hline$\% \mathrm{Fe} \mathrm{HBiO}$ & $23,0(0,00)$ & $23,0(0,00)$ & 1,000 \\
\hline$\% \mathrm{Fe} \mathrm{NH}$ Bio & $5,7(0,36)$ & $5,3(0,37)$ & 0,012 \\
\hline Energia (kcal) & $1112(32,64)$ & $1112(32,64)$ & - \\
\hline
\end{tabular}

*teste de Wilcoxon.

Média (desvio padrão).

A quantidade de FeBio de 0,53(0,06) $\mathrm{mg} \mathrm{Fe} / \mathrm{d}$, considerando-se a fortificação aumentou para $0,59(0,07)$ de 4,2 para 6,0 $\mathrm{mg} \mathrm{Fe} / \mathrm{d}$, a quantidade de FeBio aumentaria apenas $0,02 \mathrm{mg} \mathrm{Fe} / \mathrm{d}$ (Tabela 2).

$\mathrm{mg} \mathrm{Fe} / \mathrm{d}$ (Tabela 1). Elevando-se o nível de fortificação

Tabela 2. Estimativas de Fe biodisponível em dietas de creches assistenciais considerando-se dois níveis de fortificação da farinha de trigo com Fe: 4,2 mg/100g e $6,0 \mathrm{mg} / 100 \mathrm{~g}$.

\begin{tabular}{|c|c|c|c|}
\hline PARÂMETROS & $\begin{array}{c}\text { SEM } \\
\text { FORTIFICAÇÃO }\end{array}$ & $\begin{array}{c}\text { COM } \\
\text { FORTIFICAÇÃO } \\
(4,2 \mathrm{mg} \mathrm{Fe} / 100 \mathrm{~g})\end{array}$ & $\begin{array}{l}\text { COM FORTIFICAÇÃO } \\
\text { (6,0 mg Fe/100 g) }\end{array}$ \\
\hline $\mathrm{Fe} \mathrm{T} \mathrm{(mg/d)}$ & $6,2(0,76)$ & $7,7(0,87)$ & $8,3(0,95)$ \\
\hline $\mathrm{dFe}(\mathrm{mg} / 1000 \mathrm{kcal})$ & $5,8(1,25)$ & $7,7(1,65)$ & $8,5(1,84)$ \\
\hline FeBio (mg/d) & $0,53(0,06)$ & $0,59(0,07)$ & $0,61(0,07)$ \\
\hline dFeBio (mg/1000kcal) & $0,42(0,10)$ & $0,49(0,13)$ & $0,62(0,14)$ \\
\hline$\%$ FeBio & $8,6(0,38)$ & $7,6(0,40)$ & $7,3(0,42)$ \\
\hline$\% \mathrm{Fe}$ HBio & $23,0(0,00)$ & $23,0(0,00)$ & $23,0(0,00)$ \\
\hline$\%$ Fe NH Bio & $5,7(0,36)$ & $5,3(0,37)$ & $5,2(0,38)$ \\
\hline Energia (kcal) & $1112(32,64)$ & $1112(32,64)$ & $1112(32,64)$ \\
\hline
\end{tabular}

Avaliando-se as refeições individualmente, o almoço e o jantar são as refeições que mais contribuem com FeBio porque são as que tem carne em sua composição (Figuras 1 e 2). 
Figura 2. Ferro, energia, e estimativa de Fe biodisponível (FeBio) por refeição, em dietas planejadas para crianças de um a dois anos, em creches assistenciais do município de São Paulo, com e sem fortificação da farinha de trigo com ferro.

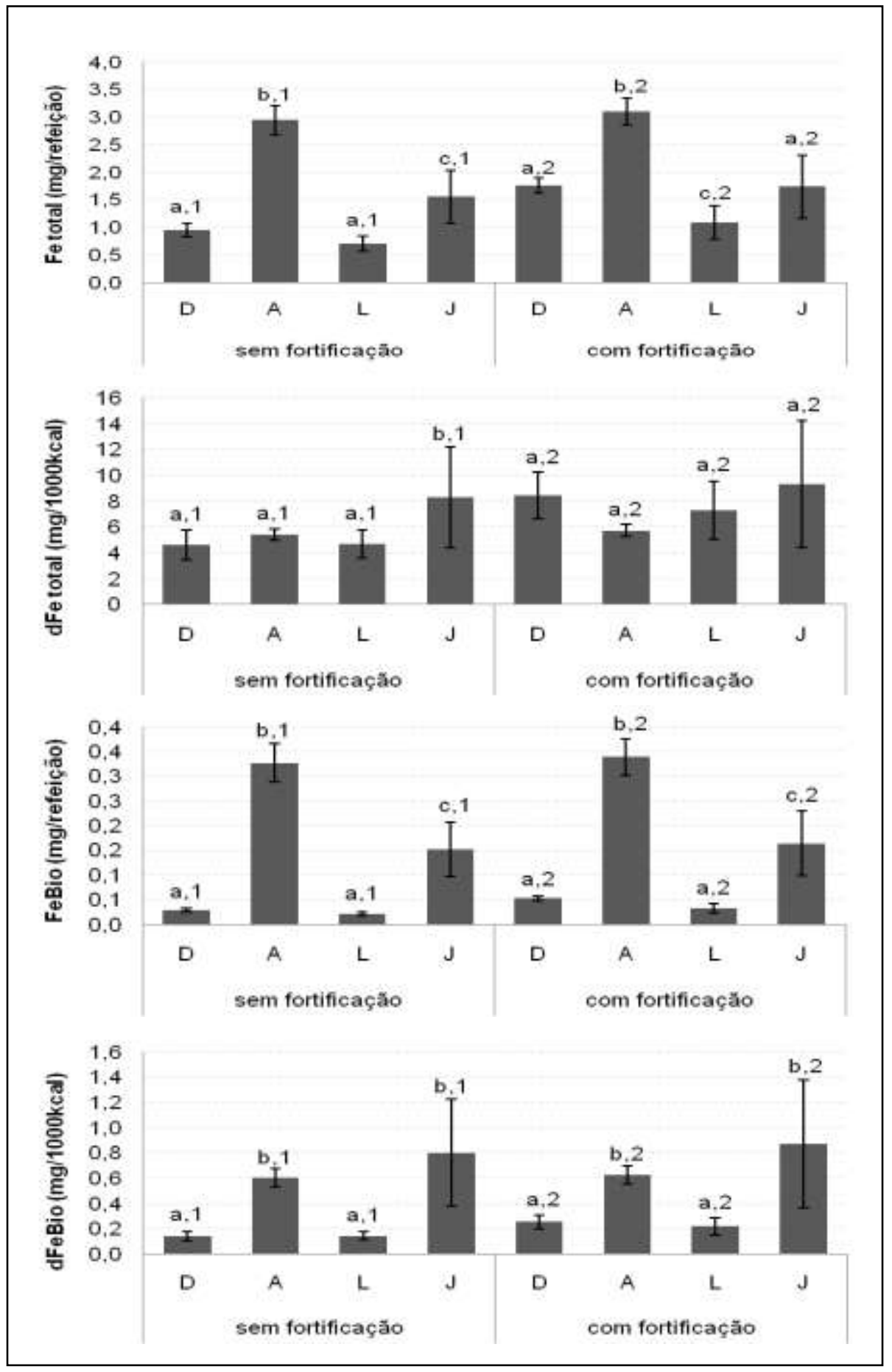

$\mathrm{D}=$ desjejum, $\mathrm{A}=$ almoço, $\mathrm{L}=$ lanche $\mathrm{J}=$ jantar.

Letras diferentes correspondem a médias diferentes no mesmo grupo pelo teste de Tukey, e números diferentes correspondem a médias diferentes entre os grupos pelo teste de Wilcoxon. 
Apesar da quantidade de FeBio não estar adequada, analisando estudos com pré-escolares que frequentam creches, observa-se tendência a redução da anemia neste grupo. (Figuras 3A e 3B).

Figura 3. Evolução da prevalência de anemia em pré-escolares: estudos em creches do município de São Paulo (A) e da região Sudeste do Brasil (B), 1999 - 2008 [21-26] [27-32].

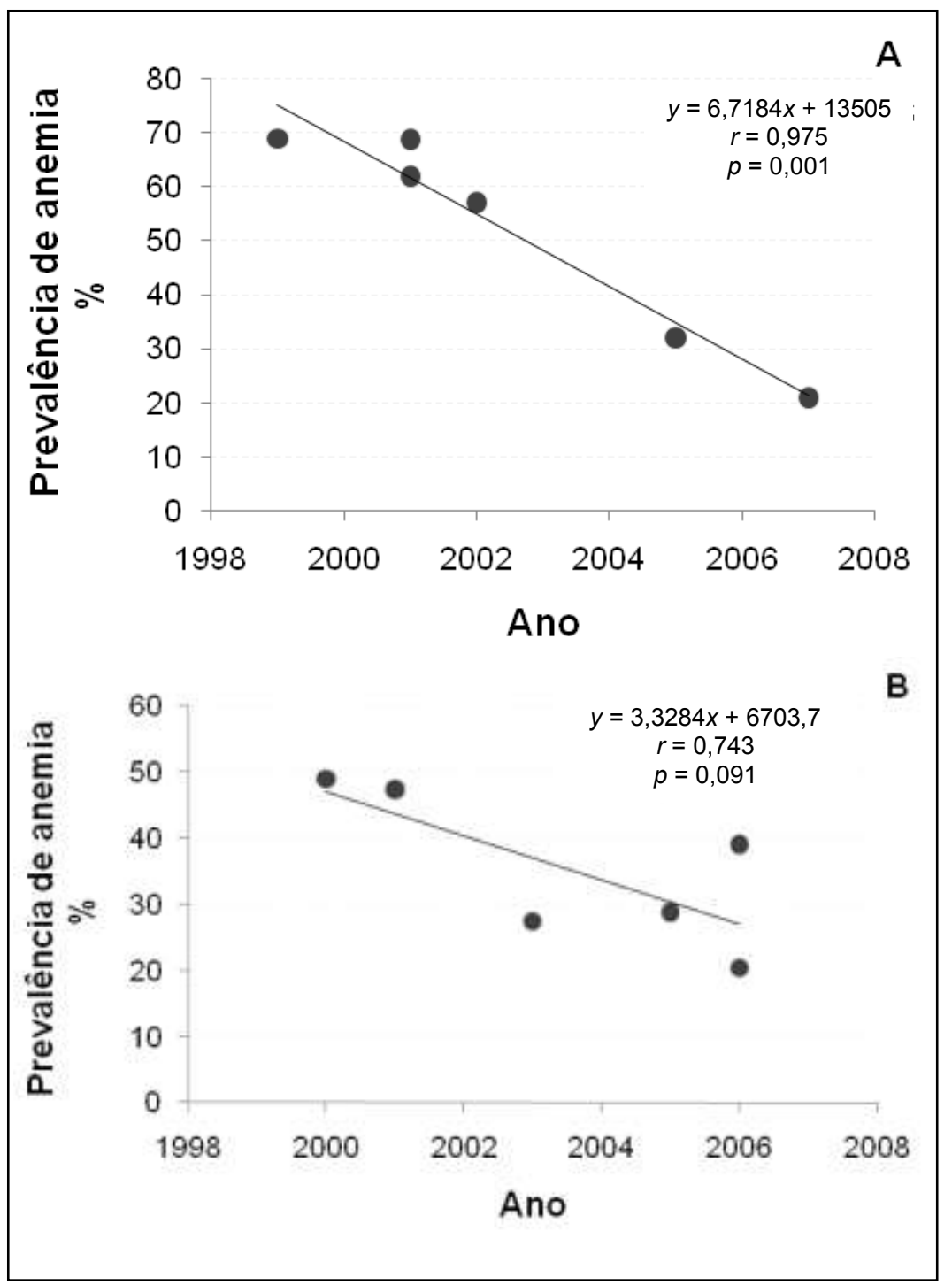




\section{Discussão}

A adequação de $\mathrm{Fe}$ na dieta é o maior determinante do status de $\mathrm{Fe}$ em crianças, sendo fortemente associada à diminuição da prevalência de anemia [18, 19]. Por outro lado, é importante ressaltar que adequação energética, nem sempre é acompanhada da adequação de nutrientes, especialmente de micronutrientes.

No presente estudo foi observada adequação energética nas dietas ofertadas às crianças, e também de $\mathrm{Fe}$, após se acrescentar à oferta diária, o total proveniente da fortificação da farinha: a média passou de $6,2(0,8) \mathrm{mg}$ $\mathrm{Fe} / \mathrm{d}$ para $7,7(0,9) \mathrm{mg} \mathrm{Fe} / \mathrm{d}$, com uma densidade de $\mathrm{Fe}$ de 7,7(1,7) mg Fe/1000 kcal, que possivelmente garantiria também para outros grupos o aporte de $\mathrm{Fe}$ dentro do recomendado (Tabela 1).

De acordo com as Referencias de Ingestão Dietética (DRI), para que a RDA de Fe fosse determinada, partiuse da necessidade média de 0,54 $\mathrm{mg} \mathrm{Fe} / \mathrm{d}$ para esse grupo. Assumindo-se a biodisponibilidade de Fe máxima da dieta como $18 \%$, a Necessidade Média Estimada (EAR) seria então $0,54 / 0,18$, ou seja, $3,0 \mathrm{mg} / \mathrm{d}$ [15].

Assim, os resultados obtidos, estimando-se o FeBio, sem incluir o Fe da fortificação, e ainda, sem considerar os fatores interferentes, mostram uma oferta média de FeBio acima da necessidade média do grupo.

Por outro lado, ao se incorporar o $\mathrm{Fe}$ da fortificação, a estimativa de oferta do mineral aumentou para 7,7 mg Fe/d, dos quais $0,59 \mathrm{mg} \mathrm{Fe} / \mathrm{d}$ são FeBio. Aumentando-se a quantidade de $\mathrm{Fe}$ nas farinhas para 6,0 $\mathrm{mg} \mathrm{Fe} / 100 \mathrm{~g}$, como o adotado por alguns países, a estimativa de FeBio atingiria $0,61 \mathrm{mg} \mathrm{Fe} / \mathrm{d}$, ou seja, se alteraria muito pouco (Tabela 2).

A interpretação desses resultados depende, então, da abordagem a ser adotada. Supondo que quando foi instituída, no final da década de 1980, a diretriz para a formulação das dietas de creche, a meta de oferta de FeBio fosse de $1,0 \mathrm{mgFe} / \mathrm{d}$, assumindo-se que a biodisponibilidade de Fe fosse de $10 \%$, e a RDA (1989) de $10 \mathrm{mg} / \mathrm{d}$ [20].

As referências de ingestão dietética de 2000 incluíram o valor do percentil 50, ou a EAR, e alteraram os valores de RDA para muitos nutrientes. Assim, foi definida a EAR de Fe de 3,0 $\mathrm{mg} / \mathrm{d}$ e a RDA (percentil $97,5)$ passou a $7,0 \mathrm{mg} / \mathrm{d}$, para crianças de um a três anos, considerando-se o máximo de $18 \%$ de biodisponibilidade ${ }^{[17]}$.

Se o objetivo era atingir o percentil 97,5 das necessidades para esse grupo, ou seja, $1,3 \mathrm{mg} / \mathrm{d}$ de FeBio (18\% dos 7,0 mg/d), de acordo com as DRI, pode-se dizer que: mesmo com uma oferta $\mathrm{de} 7,7 \mathrm{mg} / \mathrm{d}$ de Fe, maior do que a atual RDA, a meta não foi atingida (Tabela 1), e nem o seria com um aumento de $50 \%$ no nível de fortificação da farinha (de 4,2 para $6,0 \mathrm{mg}$ $\mathrm{Fe} / 100$ g) (Tabela 2). A razão é o fato de a biodisponibilidade média das dietas ofertadas nas creches ser de 9,0 \%, nem mesmo próxima do valor de $18 \%$ adotado para o cálculo da atual RDA. E aqui não estamos levando em conta as possíveis variações na concentração de $\mathrm{Fe}$ das farinhas.

Em uma primeira análise dos resultados de diversos estudos feitos com pré-escolares que freqüentam creches na cidade de São Paulo, nos últimos 10 anos, e que utilizaram a mesma metodologia de determinação da concentração de hemoglobina, o que se observa é uma tendência linear de redução na prevalência da anemia (Figura 3A) [21-26]. Tendência decrescente $(p=0,09)$, é observada quando se agrupam os estudos com crianças institucionalizadas dos outros estados da região sudeste (Figura 3B) [27-32]. E essa tendência não é aparente naqueles estudos feitos, também com préescolares, mas cuja amostragem se estende a crianças que não necessariamente freqüentam creches [33-36].

É bem conhecido o resultado do inquérito domiciliar de Monteiro, Szarfarc e Mondini [2], realizado em São Paulo, nos anos de 1984/1985 e 1995/1996, que mostrou aumento da ingestão de Fe total, de 5,4 mg $\mathrm{Fe} / \mathrm{d}$ para $7,6 \mathrm{mg} \mathrm{Fe} / \mathrm{d}$, por crianças menores de 59 meses, e que seguiu a correção do déficit energético observada na década de 80 . No entanto, apesar desse aumento de ingestão de $\mathrm{Fe}$, foi observado aumento da prevalência de anemia nas crianças (de 35,6 \% para 46,9\%), mais acentuado entre as crianças presentes nos estratos mais pobres da população, e este fato foi atribuído à menor oferta de dietas com Fe de alta biodisponibilidade para este grupo de menor poder aquisitivo.

Assunção et al. [33], em Pelotas (RS), realizaram o único estudo de impacto da fortificação das farinhas com $\mathrm{Fe}$, em pré-escolares, amostrados em seus domicílios, e observaram que não houve alteração significativa na prevalência da anemia nesse grupo (cerca de $40 \%$ ), nesse período: 2004, 2005 e 2006, e que isto pode ter se dado pelo baixo consumo de farinhas. 
Esses resultados obtidos com crianças, não necessariamente institucionalizadas, repetem-se em outros estudos: alta prevalência de anemia, associada à baixa ingestão de Fe, em torno de 5,0 mg/d [34-36].

Segundo as DRI, e sabendo que a distribuição das necessidades de Fe não é simétrica, para a avaliação da adequação dietética do mineral é recomendada a abordagem probabilística, que relaciona as ingestões individuais com a distribuição das necessidades, sendo aplicada uma função continua de probabilidade de risco para cada estimativa individual de ingestão de uma população ou grupo [37].

Assim seria interessante que fosse avaliada a distribuição da ingestão do Fe na população, definida a porcentagem de inadequação em relação à EAR, para que o nível de ingestão desejável fosse estabelecido. Ou seja, precisa-se trabalhar com o risco a ser assumido e depois definir a forma de atingir essa meta.

Esses resultados apontam, como já comentado, para uma tendência à diminuição constante da prevalência de anemia de pré-escolares que freqüentam as creches estaduais e municipais, nos últimos doze anos. Os fatores que contribuíram para esse quadro, em nosso entender, foram vários e não somente a fortificação.

O que se pode dizer é que possivelmente uma série de ações de promoção da saúde foi efetiva na redução da anemia nesse grupo de crianças institucionalizadas: a normatização de cardápios, a alteração da sistemática de compra dos alimentos (que possibilitou maior variedade dos alimentos nas refeições), a educação e orientação das mães quanto à importância do vínculo afetivo com a criança, das vacinações e da alimentação no seu desenvolvimento, e, mais recentemente, a fortificação das farinhas com Fe.

\section{Conclusões}

Considerando-se o nível de fortificação de 4,2 $\mathrm{mg} \mathrm{Fe} / 100 \mathrm{~g}$ de farinha, houve aumento de $24 \%$ no total de $\mathrm{Fe}$ oferecido nas refeições. Por outro lado, a quantidade de FeBio ofertada pouco se alterou. Para garantir as necessidades diárias de $1,26 \mathrm{mg} / \mathrm{d}$ (percentil 97,5), considerando a biodisponibilidade de 9,0\%, a recomendação diária de ferro deveria ser de $13 \mathrm{mg} / \mathrm{d}$, e não de sete $\mathrm{mg} / \mathrm{d}$. De qualquer maneira, há uma tendência de redução da prevalência de anemia na região sudeste, em crianças institucionalizadas.

\section{Referências Bibliográficas}

1. Brasil. Ministério da Saúde. Pesquisa nacional de demografia e saúde da criança e da mulher - PNDS 2006: dimensões do processo reprodutivo e da saúde da criança/Ministério da Saúde, Centro Brasileiro de Análise e Planejamento. Brasília: Ministério da Saúde, 2009. 296p.

2. Monteiro CA, Szarfarc SC, Mondini L. Tendência secular da anemia na infância na cidade de São Paulo (1984-1996). Rev Saúde Pública. 2000; 34(1):62-72.

3. Silva LSM, Giugliani ERJ, Aerts DRGC. Prevalência e determinantes de anemia em crianças de Porto Alegre, RS, Brasil. Rev Saúde Pública. 2001;35(1):66-73.

4. Jordão RE, Bernardi JLD, Barros Filho AA. Prevalência de anemia ferropriva no Brasil: uma revisão sistemática. Rev Paul Pediatr. 2009;27(1):90-98.

5. Oliveira MAA, Osório MM, Raposo MCF. Fatores socioeconômicos e dietéticos de risco para anemia em crianças de 6 a 59 meses de idade. J Pediatr. 2007;83(1):39-46.

6. WHO/UNU/UNICEF. Iron deficiency anaemia assessment, prevention and control: a guide for programme managers. Geneva, 2001. http://whqlibdoc.who.int/hq/2001/WHO_NHD_01. 3.pdf

7. Brasil. Ministério da Saúde. Agência Nacional de Vigilância Sanitária. Resolução RDC 344 de 13 de dezembro de 2002. Aprova o regulamento Técnico para fortificação das farinhas de trigo e das farinhas de milho com ferro e ácido fólico. [online] Brasília; 2002. http://e-

legis.bvs.br/leisref/public/showAct.php?id=1679.

Acesso em: 20/06/2004.

8. Pacheco ALPB, Dupret L. Creche: desenvolvimento ou sobrevivência? Psicologia USP. 2004;15(3):103-16.

9. Szarfarc SC, Monteiro CA, Meyer M, Tudisco ES, dos Reis IM. Estudo das condições de saúde das crianças do Município de São Paulo, SP (Brasil), 1984/1985. X - Consumo alimentar. Rev Saúde Pública. 1988;22:26672 .

10. Monsen ER, Balintfy JL. Calculating dietary iron bioavailability: refinement and computerization. J Am Diet Assoc. 1982;80(4):307-11.

11. Secretaria Municipal de Abastecimento. Programas de Alimentação do Município de São Paulo. São Paulo; 1996. p. 25-36. 
12. NutWin-Programa de Apoio à Nutrição [programa de computador]. Versão 1.5. São Paulo: Departamento de Informática em Saúde /UNIFESP; 2002.

13. Ramos KS. Efeito do processamento sobre a concentração de ferro heme em carnes e em pulmão bovino [dissertação]. São Paulo: Universidade de São Paulo, 1999.

14. Food and Nutrition Board. Dietary reference intakes for energy, carbohydrate, fiber, fat, fatty acids, cholesterol, protein and aminoacids (macronutrients). Washington, DC: National Academy Press; 2005. http:/ / books.nap.edu/openbook.php?record_id=1049 0\&page $=\mathrm{R} 1$

15. Fundação do Desenvolvimento da Indústria de Panificação e Confeitaria -Fundipan. Bela Vista, São Paulo: Centro de transferência de tecnologias para as indústrias de panificação e confeitaria brasileiras; 2008. Disponível

em: http://www.fundipan.org.br/. Acesso em: 10/07/08.

16. SPSS [programa de computador]. Versão 13.0 for Windows. São Paulo: SPSS Brasil; 2005.

17. Food and Nutrition Board, Institute of Medicine. Iron. In: _. Dietary reference intakes for vitamin A, vitamin $\mathrm{K}$, arsenic, boron, chromium, copper, iodine, iron, manganese, molybdenum, nickel, silicon, vanadium, and zinc. Washington, DC: National Academy Press; 2000. p. 290-393. http:/ / books.nap.edu/openbook.php?record_id=1002 6\&page $=290$

18. Wharf SG, Fox TE, Fairweather-Tait SJ, Cook JD. Factors affecting iron stores in infants 4-18 months of age. Eur J Clin. 1997;51(8):504-9.

19. Magalhães P, Ramalho RA, Colli C. Iron and vitamin A deficiencies: nutritional evaluation in pre-school children from the city of Viçosa (MG-Brazil). Nutrire. 2001;21:41-56.

20. Food and Nutrition Board, Commission on Life Sciences, National Research Council. Recommended Dietary Allowances. $10^{\text {th }}$ ed. Washington, DC: National Academy Press; 1989. http:/ / www.nap.edu/openbook.php?isbn=030904633 5

21. Ribeiro LC, Devincenzi MU, Sigulem DM. Anemia ferropriva na primeira infância: controle e prevenção com doses intermitentes de ferro quelato glicinato. Compacta Nutrição. 2001;2(2):1-22.

22. Giorgini E, Fisberg M, de Paula RA, Ferreira AM, Valle J, Braga JA. The use of sweet rolls fortified with iron bis-glycinate chelate in the prevention of iron deficiency anemia in preschool children. Arch Latinoam Nutr 2001; 51:48-53.

23. Machado EHS, Brasil AL, Palma D, Taddei JA. Condição nutricional e prevalência de anemia em crianças matriculadas em creches beneficentes. Rev Paul Pediatria 2005;23:21-6.

24. Souto TS, Oliveira MN, Casoy F, Machado EHS, Juliano Y, Gouvêa LC, Armond JE. Anemia e renda per capita familiar de crianças freqüentadoras da creche do Centro Educacional Unificado Cidade Dutra, no Município de São Paulo. Rev Paul Pediatr. 2007;25(2):161-6.

25. Bueno MB, Selem SSC, Areas JAG, Fisberg RM. Prevalência e fatores associados à anemia entre crianças atendidas em creches públicas de São Paulo. Rev Bras Epidemiol. 2006;9(4):46270.

26. Costa CA, Machado EHS, Colli C, Latorre WC, Szarfarc SC. Anemia em pré-escolares atendidos em creches de São Paulo (SP): perspectivas decorrentes da fortificação das farinhas de trigo e de milho. Nutrire. 2009; 34(1):56-74.

27. Lamounier JA, Bracarense B, Chamone BC, Campos CT, Rocha F, Hortmann HC et al. Prevalência de anemia ferropriva e parasitose intestinal em crianças de 0 a 6 anos no município de Carrancas, MG. Anais do III Encontro de Extensão e Primeira Semana do Conhecimento da UFMG: Belo Horizonte, MG; 2000.

28. Almeida AP, Zandonade E, Abrantes MM, Lamounier JA. Deficiência de ferro e anemia em crianças de Vitória, ES. Pediatria. 2004;26:140-50.

29. Matta IEA, Veiga GV, Baião MR, Santos, MMAS, Luiz RR. Anemia em crianças menores de 5 anos que freqüentam creches públicas do Município do Rio de Janeiro, Brasil. Rev Bras Saúde Matern Infant. 2005;5:349-57.

30. Rocha DS, Lamounier JA, Capanema FD, Franceschini SCC, Norton RC, Costa AB et al. Estado nutricional e prevalência de anemia em crianças que freqüentam creches em Belo Horizonte, Minas Gerais. Rev Paul Pediatr. 2008;26(1):6-13.

31. Bagni UV, Baião MR, Santos MMAS, Luiz RR, da Veiga GV. Efeito da fortificação semanal do arroz com ferro quelato sobre a freqüência de anemia $e$ concentração de hemoglobina em crianças de creches municipais do Rio de Janeiro, Brasil. Cad Saúde Pública. 2009;25(2):291-302.

32. Santos JN, Rates SPM, Lemos SM, Lamounier JA. Anemia em crianças de uma creche pública e as repercussões sobre o desenvolvimento de linguagem. Rev Paul Pediatr. 2009;27(1):67-73. 
33. Assunção MCF, Santos IS, Barros, AJD, Gigante DP, Victora CG. Efeito da fortificação de alimentos com ferro sobre anemia em crianças: um estudo de revisão. Rev Saúde Pública 2007;41(4):1-10.

34. Compri PC, Cury MCFS, Novo NF, Juliano Y, Sigulem DM. Variáveis maternas e infantis associadas à ocorrência de anemia em crianças nos serviços de atenção básico em São Paulo. Rev Paul Pediatr. 2007;25(4):349-54.
35. Vitolo MR, Bortolini GA. Iron bioavailability as a protective factor against anemia among children aged 12 to 16 months. J Pediatr. 2007;83(1):33-8.

36. Oliveira MAA, Osório MM, Raposo MCF. Socioeconomic and dietary risk factors for anemia in children aged 6 to 59 months. J Pediatr. 2007;83(1):3946.

37. Food and Nutrition Board, institute of Medicine. Dietary reference intakes: applications in dietary planning. Washington, DC: National Academy Press; 2003. 